\title{
Impaired Muscarinic Endothelium-dependent Relaxation and Cyclic Guanosine 5'-Monophosphate Formation in Atherosclerotic Human Coronary Artery and Rabbit Aorta
}

\author{
Claus Bossaller, Gabriel B. Habib, Hideo Yamamoto, Carolyn Williams, Sharon Wells, and Philip D. Henry \\ Section of Cardiology, Department of Medicine, Baylor College of Medicine, and The Methodist Hospital, Houston, Texas 77030
}

\begin{abstract}
The dependence of vascular relaxation on an intact endothelium and the relationship between relaxation and cyclic GMP accumulation were determined in coronary arteries isolated from cardiac transplantation patients with or without coronary atherosclerosis. In nonatherosclerotic arteries, the endothelium-dependent agent acetylcholine produced concentration-related relaxations. In atherosclerotic arteries, endothelium-dependent relaxations were abolished with acetylcholine, partly suppressed with substance $P$ and histamine, and completely preserved with the ionophore A23187. In these arteries, the endothelium-independent agent nitroglycerin remained fully active. Accumulation of cyclic GMP in atherosclerotic strips was suppressed with acetylcholine but unattenuated with A23187 and nitroglycerin. In aortas from rabbits with diet-induced atherosclerosis, there was likewise an impaired cholinergic relaxation and cyclic GMP accumulation in the presence of preserved responses to A23187 and nitroglycerin. The results demonstrate that impaired cholinergic responses in atherosclerotic arteries reflect a muscarinic defect and not an inability of endothelium to release endothelial factor or smooth muscle to respond to it.
\end{abstract}

\section{Introduction}

In a previous study we have demonstrated that vascular relaxation in response to acetylcholine is impaired in cholesterol-fed rabbits (1). Experiments with isolated vessels have shown that acetylcholine exerts its relaxing effects by stimulating the release of a relaxing principle from endothelial cells (endothelium-derived relaxing factor [EDRF] ${ }^{1}$ (2). Ultrastructural studies of rabbit aortas with impaired cholinergic relaxation exhibit an intact endothelial cell lining and only modest changes in endothelial cell structure. This indicates that the cholinergic defect is not related to atherosclerotic endothelial denudation (1).

The present study was designed to determine whether impaired cholinergic relaxation is also demonstrable in atheroscle-

Address correspondence to Dr. Henry, Prof. of Medicine, Section of Cardiology, Baylor College of Medicine, 6535 Fannin, Suite F-905, Houston, TX 77030. 1986.

Received for publication 27 May 1986 and in revised form 18 August

1. Abbreviations used in this paper: EDRF, endothelium-derived relaxing factor; $\mathrm{PGF}_{2 \alpha}$, prostaglandin $\mathrm{F}_{2 \alpha}$.

J. Clin. Invest.

(c) The American Society for Clinical Investigation, Inc. 0021-9738/87/01/0170/05 \$1.00

Volume 79, January $1987,170-174$ rotic human coronary arteries. In addition, we wanted to ascertain whether the defective endothelium-dependent relaxation is selective for acetylcholine, or whether the unresponsiveness represents a generalized failure of endothelium to release EDRF or smooth muscle to respond to it.

\section{Methods}

\section{Experiments with human coronary arteries}

Patient characteristics. The patients consisted of 22 subjects undergoing cardiac transplantation at The Methodist Hospital and at The Texas Heart Institute. There were 20 males and 2 females, who ranged in age from 12 to $62 \mathrm{yr}$ (median age $41 \pm 3$ ). The preoperative diagnoses were ischemic heart disease in 14 , and cardiomyopathy in 8 patients. Drug treatment during the $2 \mathrm{wk}$ before the operations included furosemide (20 patients), captopril (17 patients), digoxin (16 patients), and nitrates (16 patients).

\section{Pharmacological measurements}

The recipient's heart was collected immediately after its excision and immersed in room temperature oxygenated Krebs-Henseleit buffer of the following composition (millimolars): $\mathrm{NaCl}, 118 ; \mathrm{KCL}, 4.0 ; \mathrm{CaCl}_{2}$, $1.5 ; \mathrm{NaH}_{2} \mathrm{PO}_{4}, 1.2 ; \mathrm{MgSO}_{4}, 1.2 ; \mathrm{NaHCO}_{3}, 25 ;$ and dextrose, 5. 4-5-mm wide rings were cut from the proximal $4 \mathrm{~cm}$ of the left circumflex and right coronary arteries, avoiding sites of major arterial ramifications. The rings were opened and mounted as transverse strips in an organ bath filled with Krebs-Henseleit solution equilibrated at $37^{\circ} \mathrm{C}$ with a $95 \%$ $\mathrm{O}_{2} / 5 \% \mathrm{CO}_{2}$ gas mixture. One end of the strip was attached to the bottom of the chamber, the other to a Statham 4C-2 force transducer, which was connected to a Gould amplifier/recorder system. The strips were allowed to stabilize for $60 \mathrm{~min}$ under a preload of $2 \mathrm{~g}$. They were then contracted with $10 \mu \mathrm{M}$ PGF $_{2 \alpha}$ to effect an increase in tone of 2-3 g and subsequently relaxed by the cumulative addition of acetylcholine ( $1 \mathrm{nM}-$ $1 \mu \mathrm{M})$, calcium ionophore A23187 (1 nM-1 $\mu \mathrm{M})$, substance $\mathrm{P}(0.01-10$ $\mathrm{nM})$, histamine (1 nM-1 $\mu \mathrm{M})$, or nitroglycerin $(1 \mu \mathrm{M})$. Concentrationeffect relations were determined for each agonist, but the data were not subjected to receptor-pharmacological analyses, since the variable oscillatory tone typical of human coronary arteries renders such analyses difficult. In this study, we have plotted data for maximal tone (peaks of oscillations), minimal tone (troughs of oscillations), and in some instances for mean tone (time integral/time). The trends of the results were very similar with the three different data sets for the agonists tested. For simplicity, we present here values for minimal tone (troughs), mainly because the variance was smallest with this data set. In some experiments, the dose-response experiments were repeated with or without prior de-endothelialization of the strips. The endothelium was removed mechanically by rubbing the intimal surface with a smooth stick as described by Furchgott et al. (2). Quantitative scanning electronmicroscopic studies in our laboratories have demonstrated the completeness of the de-endothelialization achieved by this method (1).

\section{Morphologic studies}

At the end of the pharmacological experiments the strips were fixed in $10 \%$ formalin without dismounting them from their isometric attachments. The fixed tissue was embedded in paraffin and histologic sections 
were stained with hematoxylin-eosin and Verhoeff-Van Gieson stain. In this study, arteries were assigned to three groups according to structural criteria derived from the description of human coronary arteries of young adults $(3,4)$. The classification was designed to include patients with arteries showing either no atherosclerotic changes or definite atherosclerotic lesions. Criteria for the three groups were as follows:

Group I. (a) maximal ratio intimal to medial thickness $<1.0$; (b) only occasional subendothelial clusters of foam cells; (c) no intimal accumulation of smooth muscle cells and collagen; $(d)$ no lipid-laden smooth muscle cells in intima; and $(e)$ no extracellular intimal debris (lipid particles, cholesterol crystals, apatite crystals, membranous organelle fragments). In this study, the term "nonsclerotic strip" refers to arteries conforming to the criteria of group I in conjunction with a preoperative serum cholesterol concentration of $<200 \mathrm{mg} / \mathrm{dl}$.

Group II. Arteries with mild or disputable changes of atherosclerosis and with structural changes conforming neither to those of groups I or III were assigned to this group. Such changes included fatty streaks, intimal thickening, and fibrous lesions lacking a core of debris. Since such arteries may or may not be classified as atherosclerotic, we have excluded results obtained with group II arteries.

Group III. (a) maximal ratio intimal to medial thickness $>2$ (range 2.0 to $4.7 ;(b)$ lesions consisting of caps of dense fibrous tissue (collagen) surrounding cores of lipid-rich and calicific debris; $(c)$ capillary-sized vessels surrounding the fibrotic lesions (plaque vascularization); $(d)$ preserved endothelial cell layer overlying the fibrous lesions, i.e., absence of ulceration; and ( $e$ ) absence of fresh thrombi (fibrin). Therefore, group III arteries contained complicated lesions without intimal surface changes including denudation and thrombosis. In this study, the term "sclerotic strips" corresponded to arteries conforming to the criteria of group III.

\section{Measurement of cyclic GMP}

12-15-mm long arterial segments were cut into three rings of equal width. The two outer rings were used for mechanical experiments as described above, and the middle ring was used for the measurement of cyclic GMP. Opened middle rings were mounted isometrically as transverse strips in a myograph containing Kreb's buffer (as for mechanical experiments), and subsequently contracted by the addition of prostaglandin $\mathrm{F}_{2 \alpha}\left(\mathrm{PGF}_{2 \alpha}\right)$ (final concentration, $10 \mu \mathrm{M}$ ). Concentrated drugs in Kreb's buffer were added directly to the bath fluid which was being stirred with a magnetic bar to effect rapid mixing. At selected intervals after drug admixture, the strips were freeze-clamped with a Wollenberger clamp cooled to liquid nitrogen temperature. The tissue was subsequently homogenized in a glass/glass homogenizer in ice-cold 6\% TCA. The homogenates were centrifuged at $1,700 \mathrm{~g}$ for $5 \mathrm{~min}$ at $4^{\circ} \mathrm{C}$. Precipitates were used for protein determination by the method of Lowry et al. (5) with bovine serum albumin as the standard. Supernatant fractions were extracted three times with ether and the aqueous phase was lyophilized. The residue was dissolved in $50 \mathrm{mM}$ sodium acetate buffer and cyclic GMP was measured by a commercial radioimmunoassay (New England Nuclear, Boston, MA).

\section{Experiments with isolated rabbit aortas}

32 male New Zealand white rabbits weighing between 2.5 and $3.1 \mathrm{~kg}$ were placed at random either on $1 \%$ cholesterol pellets or on standard rabbit pellets (both supplied by ICN Nutritional Biochemicals, Cleveland, $\mathrm{OH})$. At the end of a 10-wk diet period, the rabbits were killed under pentobarbital anesthesia $(30 \mathrm{mg} / \mathrm{kg}$ i.v. $)$ after a $24-\mathrm{h}$ fast. The thoracic aorta was promptly excised and immersed in Krebs-Henseleit buffer at $21^{\circ} \mathrm{C}$. The isolated aorta was cleaned of perivascular tissue and 4-mm wide rings were cut from the mid-descending thoracic aorta. The rings were opened and the transverse strips mounted in an organ bath for the recording of isometric tension. After $60 \mathrm{~min}$ of equilibration, the rings were contracted with $1 \mu \mathrm{M}$ phenylephrine and then relaxed by the cumulative addition of acetylcholine, A23187, or nitroglycerin (final concentrations for all drugs, $1 \mathrm{nM}$ to $1 \mu \mathrm{M}$ ). Cyclic GMP levels in aortic strips were measured as described for the human coronary arteries, except that the vascular preparations were precontracted with $1 \mu \mathrm{M}$ phenylephrine.

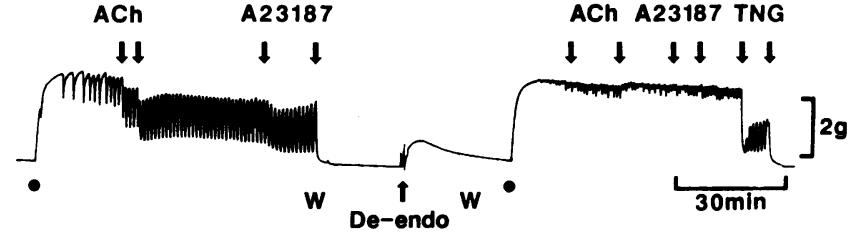

Figure 1. Effects of de-endothelialization on the responsiveness of isolated nonsclerotic human coronary artery to vasodilators. (Solid circles) Contractions elicited by $10 \mu \mathrm{M} \mathrm{PGF}_{2 \alpha}$. W, wash; De-endo, deendothelialization. Before de-endo: acetylcholine (ACh), 0.1 and 1.0 $\mu \mathrm{M}$, produces dose-dependent decreases in oscillating tone; $\mathrm{A} 23187$, 0.1 and $1.0 \mu \mathrm{M}$, results in additional reductions in tone. The higher A23187 concentration also abolishes rhythmic activity. After de-endo: $\mathrm{ACh}, 0.1$ and $1.0 \mu \mathrm{M}$, and $\mathrm{A} 23187,0.1$ and $1.0 \mu \mathrm{M}$, become ineffective, although nitroglycerin (TNG), 0.1 and $1.0 \mu \mathrm{M}$, remains potent.

\section{Data analysis and statistics}

The significance of the difference between group means was analyzed by Wilcoxon's rank test or, with normally distributed data, by $t$ tests for unpaired samples. $P$ values $<0.05$ were taken as statistically significant.

\section{Results}

Human coronary arteries. A representative tracing of an experiment illustrating the dependence of vascular relaxation on an intact endothelium is shown in Fig. 1. In the nonsclerotic coronary artery, acetylcholine and A23187 were effective in evoking relaxation in the presence of an intact endothelium. $1 \mu \mathrm{M}$ A23187 effected nearly complete relaxation. After de-endothe-
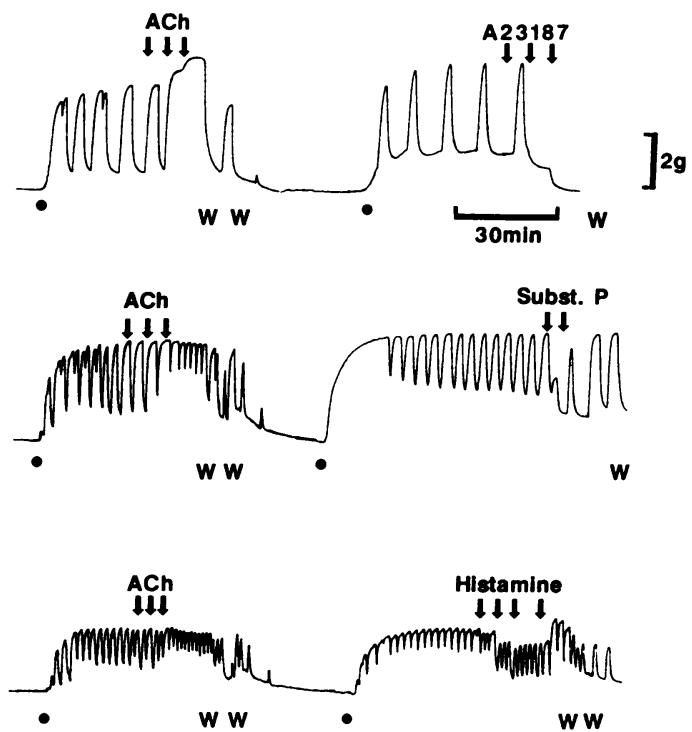

Figure 2. Effects of endothelium-dependent vasodilators on isolated atherosclerotic human coronary artery. (Solid circles) Contractions elicited by $10 \mu \mathrm{M} \mathrm{PGF}_{2 \alpha}$. W, wash. Arrows on top of the three tracings correspond to the cumulative addition of drug. (Top tracing) Acethylcholine (ACh), 0.01, 0.1, and $1.0 \mu \mathrm{M}$, first fails to relax, and then contracts artery. In contrast, $A 23187,0.01,0.1$, and $1.0 \mu \mathrm{M}$, produces complete relaxation at the highest concentration. (Middle tracing) Acetylcholine ( $\mathrm{ACh}), 0.01,0.1$, and $1.0 \mu \mathrm{M}$, produces no reduction in tone. In contrast, substance P (Subst. P), 0.1 and $1.0 \mathrm{nM}$, decreased rhythmic tone. (Bottom tracing) Acetylcholine (ACh), 0.01, 0.1 , and $1.0 \mu \mathrm{M}$, produces no reduction in tone, whereas histamine, $0.01,0.1,1.0$, and $10.0 \mu \mathrm{M}$, effects partial relaxations. 
Table I. Relaxation of Human Coronary Artery before and after De-endothelialization

\begin{tabular}{llllll}
\hline & \multicolumn{2}{l}{ Nonsclerotic* } & & \multicolumn{2}{c}{ Sclerotic* } \\
\cline { 2 - 3 } \cline { 6 - 6 } & +ENDO & -ENDO & & +ENDO & -ENDO \\
\hline Acetylcholine $(1 \mu \mathrm{M})$ & $54.3 \pm 9.2^{\ddagger}$ & 0.0 & & $2.1 \pm 0.1^{8}$ & 0.0 \\
A23187 $(1 \mu \mathrm{M})$ & $84.9 \pm 2.9$ & $6.4 \pm 0.9$ & & $86.9 \pm 2.7$ & $9.2 \pm 0.5$ \\
Substance P $(1 \mathrm{nM})$ & $62.1 \pm 6.1$ & $2.4 \pm 0.4$ & & $35.0 \pm 8.3^{\S}$ & $1.2 \pm 0.2$ \\
Histamine $(1 \mu \mathrm{M})$ & $68.0 \pm 8.1$ & 0.0 & & $40.4 \pm 5.2^{8}$ & 0.0 \\
Nitroglycerin $(1 \mu \mathrm{M})$ & $95.4 \pm 2.4$ & $94.8 \pm 2.4$ & & $90.3 \pm 2.2$ & $88.7 \pm 1.9$
\end{tabular}

"The terms "nonsclerotic" (5 patients) and "sclerotic" (15 patients) are defined under Methods. +ENDO and-ENDO refer to before and after de-endothelialization.

${ }^{\ddagger}$ Values are means \pm SE ( $n=13$ to 24 strips) and represent percent decreases in $\mathrm{PGF}_{2 \alpha}(10 \mu \mathrm{M})$-induced constrictor tone. The drug concentrations used were those producing maximal decreases in tone.

Values significantly different $(P<0.01)$ compared with corresponding values in nonsclerotic arteries.

lialization, the endothelium-dependent agents acetylcholine and A23187 became ineffective, whereas the endothelium-independent agent nitroglycerin still produced complete relaxation. In the sclerotic strip without de-endothelialization, the responsiveness to acetylcholine was virtually lost, although the endothelium-dependent ionophore was still quite potent, with $1 \mu \mathrm{M}$ A23187 producing virtually complete relaxation (Fig. 2). Therefore, the results with A23187 indicate that the atherosclerotic endothelium was still capable of releasing EDRF, and that the thickened atherosclerotic intima did not represent a barrier for the factor to reach and relax smooth muscle in the media.

To evaluate to which extent the endothelial defect was selective for acetylcholine, additional experiments with other endothelium-dependent agents were performed. Fig. 2 illustrates that strips with unresponsiveness to acetylcholine were still capable of relaxing in response to substance $\mathbf{P}$ and histamine, although the responses were significantly attenuated. De-endothe- lialization abolished the relaxations in response to substance $\mathbf{P}$ and histamine (Table I). Fig. 3 summarizes the results of concentration-response experiments with acetylcholine, A23187, substance $P$, and histamine.

Table II shows the results of the measurements of cyclic GMP after stimulation with acetylcholine, A23187, or nitroglycerin. Before stimulation, cyclic GMP levels did not differ significantly between nonsclerotic and sclerotic strips. In both nonsclerotic and sclerotic strips, increases in cyclic GMP levels after stimulation with the three drugs were significantly higher at $60 \mathrm{~s}$ than at $30 \mathrm{~s}(P<0.05)$, and those at $90 \mathrm{~s}$ did not differ significantly $(P<0.3)$. On the basis of these time course observations, 60 -s values were taken to represent peak increases. Although 60 -s values in nonsclerotic and sclerotic arteries were similar for A23187 and nitroglycerin, they differed for acetylcholine, with sclerotic arteries exhibiting significantly lower values than nonsclerotic strips (Table II).

Light microscopic examination of the arterial strips revealed complicated plaques conforming to the structural criteria of group III in the majority of the patients (15 of 22). In only five patients (all with cardiomyopathies) could atherosclerotic changes not be demonstrated (group I). The arteries from two patients showed minor fibrotic changes (group II) and were eliminated from the study (see Methods). Strips not subjected to de-endothelialization exhibited in no instance areas of endothelial denudation. Strips subjected to mechanical de-endothelialization exhibited denudations involving $>90 \%$ of the intimal surface area and only occasional clusters of mostly damaged endothelial cells.

Rabbit aortas. The results of the mechanical experiments with isolated rabbit aortas are summarized in Table III. Increases in tension evoked by phenylephrine were similar in the two groups. On the other hand, relaxations in response to acetylcholine were impaired in the atherosclerotic strips, although relaxations with A23187 and nitroglycerin were largely preserved. Therefore, as in the human coronary artery, there appeared to be a selective cholinergic impairment. The selective impairment with acetylcholine was also reflected in a selective suppression
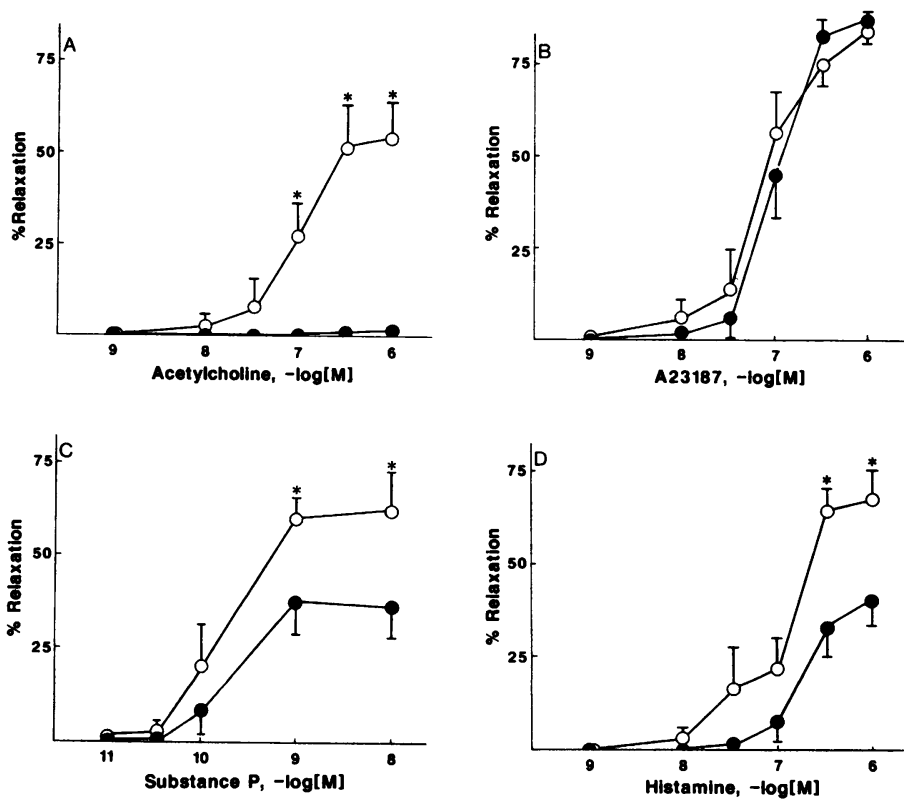

Figure 3. Concentration-response curves for human coronary arteries with the $(A)$ endothelium-dependent vasodilators acetylcholine, $(B) \mathrm{A} 23187,(C)$ substance $\mathrm{P}$, and $(D)$ histamine. Data points with vertical bars (1 SE) represent mean values derived from 14 to 24 experiments, and express percent reductions in constrictor tone produced by $10 \mu \mathrm{M} \mathrm{PGF}_{2 \alpha}$. (Asterisks) Values in sclerotic arteries (solid circles) differed significantly $(P<0.01)$ from those in the nonsclerotic arteries (open circles). 
Table II. Cyclic GMP Formation in Human Coronary Artery

\begin{tabular}{llr}
\hline & Nonsclerotic $^{*}$ & \multicolumn{1}{c}{ Sclerotic* $^{*}$} \\
\hline 60 min after PGF $2 \alpha(1 \mu \mathrm{M})$ & $188 \pm 23^{\ddagger}$ & $210 \pm 34$ \\
60 s after acetylcholine $(1 \mu \mathrm{M})$ & $452 \pm 49$ & $249 \pm 41^{\S}$ \\
60 s after A23187 $(1 \mu \mathrm{M})$ & $982 \pm 110$ & $886 \pm 102$ \\
60 s after nitroglycerin $(1 \mu \mathrm{M})$ & $989 \pm 107$ & $1,101 \pm 209$ \\
\hline
\end{tabular}

* The terms nonsclerotic (two patients) and sclerotic (four patients) are defined under Methods.

¥ Values are expressed in picomoles per gram protein and represent means $\pm \mathrm{SE}$ ( $n=6$ to eight strips).

8 Value significantly different $(P<0.01)$ compared with corresponding value of nonsclerotic arteries. The three vasodilators were added $1 \mathrm{~h}$ after incubation in buffer containing $10 \mu \mathrm{M} \mathrm{PGF}_{2 \alpha}$.

of cyclic GMP accumulation (Table III). Time course experiments showed that 30-s values corresponded in all groups of strips to maximal increases in cyclic GMP, i.e., values at $15 \mathrm{~s}$ were significantly lower $(P<0.05)$, and those at $60 \mathrm{~s}$ did not differ significantly $(P>0.5)$.

\section{Discussion}

Results of this study demonstrate that the human coronary artery, as that of other large mammals, relaxes in response to acetylcholine via an endothelium-dependent mechanism. This finding appears to be in conflict with earlier reports emphasizing that the human coronary artery is unlike that of other mammals and lacks the phenomenon of muscarinic endothelium-dependent relaxation $(6,7)$. We believe that this apparent disparity reflects the fact that previous experiments were performed with either diseased coronary arteries or arteries prepared without sufficient precautions to protect endothelial function. Pilot experiments in our laboratories have repeatedly demonstrated that cadaver coronary arteries cannot be used for the study of endothelium-dependent relaxation. Also, it is important to immerse the heart of patients undergoing cardiac transplantation immediately after excision to avoid endothelial damage related to air exposure (8). One difficulty is that human coronary arteries are often diseased, even when isolated from cardiomyopathic patients preoperatively thought to have little or no coronary atherosclerosis. Preservation of cholinergic relaxation is clearly not only an age-dependent mechanism, since one of our patients who exhibited excellent cholinergic relaxation was 54-yr-old. Therefore, there is no indication that the responsiveness of the human coronary to acetylcholine is anomalous compared with that of other large mammals.

Our structural studies suggest that the loss of endotheliumdependent relaxation is related to atherosclerosis, since arterial strips free of atherosclerotic changes relaxed promptly in response to acetylcholine. This conclusion is supported by our experimental studies with cholesterol-fed rabbits which demonstrate that cholinergic relaxation of aortas from these rabbits is impaired.

Of considerable interest was the observation that other agents, in particular the calcium ionophore A23187, were still potent in relaxing the coronary artery and the rabbit aorta when acetylcholine was ineffective. This suggests that the defect has at least partial selectivity for acetylcholine and does not represent an inability of endothelial cells to produce and release EDRF, or an impediment of the factor to diffuse through the thickened intima, or a refractoriness of smooth muscle to EDRF. Since atherosclerosis has been previously shown to alter surface receptor functions (9), one might argue that maintained responsiveness to the ionophore reflects the fact that this agent bypasses actions mediated by membrane-specific mechanisms. The partially maintained relaxations with substance $P$ and histamine in the presence of refractoriness to acetylcholine, however, demonstrate that endothelial membranes exposed to appropriate nonmuscarinic stimuli are still capable of transmitting signals for the release of EDRF.

There is considerable evidence that EDRF acts on smooth muscle by stimulating the accumulation of cyclic GMP, but the detailed molecular mechanism responsible for muscular relaxation via the guanylate cyclase system is still incompletely understood $(10,11)$. Of interest is that other vasodilators such as nitrates and atrial natriuretic factor likewise stimulate guanylate cyclase in smooth muscle, but their action appears to be direct on smooth muscle and independent of endothelial cells. Our finding that the atherosclerotic cholinergic impairment was as-

Table III. Relaxation and Cyclic GMP Formation in Aortas from Rabbits Fed a Standard or 1\% Cholesterol Diet

\begin{tabular}{lrrr}
\hline & Standard diet & $1 \%$ Cholesterol diet \\
\hline Pharmacological measurements & & & \\
Phenylephrine $(1 \mu \mathrm{M})$ contraction $(\mathrm{g})$ & $2.3 \pm 0.1$ & $(n=44)$ & $2.4 \pm 0.1 \quad(n=52)$ \\
\% Relaxation with acetylcholine $(1 \mu \mathrm{M})^{*}$ & $69.5 \pm 3.6 \quad(n=32)$ & $34.0 \pm 2.9 \quad(n=40)^{8}$ \\
\% Relaxation with A23187 $(1 \mu \mathrm{M})^{*}$ & $73.9 \pm 2.2 \quad(n=32)$ & $69.9 \pm 1.8 \quad(n=40)$ \\
\% Relaxation with nitroglycerin $(1 \mu \mathrm{M})^{*}$ & $91.2 \pm 2.8 \quad(n=12)$ & $87.3 \pm 2.3 \quad(n=12)$ \\
Cyclic-GMP measurements & & & \\
60 min after phenylephrine $(1 \mu \mathrm{M})$ & $193 \pm 37 \quad(n=9)$ & $73 \pm 11 \quad(n=10)^{8}$ \\
30 s after acetylcholine $(1 \mu \mathrm{M})$ & $734 \pm 40 \quad(n=12)$ & $374 \pm 46 \quad(n=18)^{8}$ \\
30 s after A23187 $(1 \mu \mathrm{M})$ & $1,093 \pm 163 \quad(n=12)$ & $1,221 \pm 278 \quad(n=7)$ \\
30 s after nitroglycerin $(1 \mu \mathrm{M})$ & $1,257 \pm 229 \quad(n=13)$ & $1,317 \pm 305 \quad(n=10)$ \\
& & & \\
\hline
\end{tabular}

* Values are means $\pm \mathrm{SE}$ and represent maximal relaxations expressed as a percent of the contractions produced by $1 \mu \mathrm{M}$ phenylephrine. ${ }^{\ddagger}$ Values are expressed in picomoles per gram protein and represent means \pm SE. ${ }^{\delta}$ Values significantly different $(P<0.01)$ compared with corresponding values in the standard diet group. The three vasodilators were added $1 \mathrm{~h}$ after incubation in buffer containing $1 \mu \mathrm{M}$ phenylephrine. 
sociated with decreased cyclic GMP accumulation is in agreement with the concept that muscarinic relaxation depends upon the guanylate cyclase system. That cyclic GMP accumulation was unimpaired with the indirect stimulation with $A 23187$ or with the direct stimulation with nitroglycerin indicates that atherosclerotic smooth muscle retains a responsive receptor mechanism for EDRF and a preserved guanylate cyclase activity.

There is increasing evidence that endothelium-dependent relaxation may play an important role in determining the vasodilator reserve of arterial beds (12). Therefore, we believe that the demonstration of an impaired endothelium-dependent mechanism might have important implications for pathophysiological processes requiring vasodilation. Although nonneurogenic cholinergic stimulation may seem to represent an artificial (pharmacological) event, it is of interest that endothelial cells appear to have the capacity to synthesize acetylcholine (13). Clinical reports indicate that some patients suffering from angina respond to cholinergic stimulation with coronary constriction (14-16). This may be explained on the basis of an impaired endothelium-dependent relaxation, an impairment that leaves the direct constrictive effects of acetylcholine on smooth muscle unopposed (2).

\section{Acknowledgments}

The authors wish to thank Dr. Kearney, Department of Pathology, for the light microscopic evaluation of the arteries. We are indebted to Drs. Frazier and Noon for their support in procuring the human arteries. Our appreciation is also extended to Ms. W. Kearns for excellent secretarial assistance.

This research was supported in part by grants from the National Heart, Lung and Blood Institute (HL31487, HL31488, and HL27341), and by a fellowship grant from the Deutsche Forschungsgemeinschaft (B0771/1-1).

\section{References}

1. Habib, J., C. Bossaller, S. Wells, C. Williams, J. Morrisett, and P. D. Henry. 1986. Preservation of endothelium-dependent vascular relaxation in cholesterol-fed rabbit by treatment with the calcium blocker PN 200110. Circ. Res. 58:305-309.

2. Furchgott, R. F., P. D. Cherry, J. V. Zawadski, and D. Jothianandan. 1984. Endothelial cells as mediators of vasodilation of arteries. J. Cardiovasc. Pharmacol. 6:S336-S343.

3. Stary, H. C. 1983. Structure and ultrastructure of the coronary artery intima in children and young adults up to age 29. In Atherosclerosis. VI. F. G. Schettler, A. M. Gotto, G. Middelhoff, A. Y. Habenicht, and K. R. Jurutka, editors. Springer Verlag, Berlin, Heidelberg, New York. 82-86.

4. Stary, H. C. 1983. Macrophages in coronary artery and aortic intima and in atherosclerotic lesions of children and young adults up to age 29. In Atherosclerosis. VI. F. G. Schettler, A. M. Gotto, G. Middelhoff, A. Y. Habenicht, and K. R. Jurutka, editors. Springer Verlag, Berlin, Heidelberg, New York. 462-466.

5. Lowry, O. H., N. Y. Rosebrough, A. L. Farr, and A. J. Randall. 1951. Protein measurements with Folin phenol reagent. J. Biol. Chem. 193:265-275.

6. Kalsner, S. 1984. Cholinergic mechanisms in human coronary artery preparations: implications of species differences. J. Physiol. 358: 509-526.

7. Toda, N. 1983. Isolated human coronary arteries in response to vasoconstrictor substances. Am. J. Physiol. 245:H937-H941.

8. Fishman, Y. A., G. B. Ryan, and M. J. Karnovsky. 1975. Endothelial regeneration in the rat carotid artery and the significance of endothelial denudation in the pathogenesis of myointimal thickening. Lab. Invest. 32:339-351.

9. Henry, P. D., and M. Yokoyama. 1980. Supersensitivity of atherosclerotic rabbit aorta to ergonovine: mediation by a serotonergic mechanism. J. Clin. Invest. 66:306-313.

10. Murad, F., R. M. Rapoport, and R. Fiscus. 1985. Role of cyclicGMP in relaxations of vascular smooth muscle. J. Cardiovasc. Pharmacol. 7:111-118.

11. Ignarro, L. J., and P. J. Kadowitz. 1985. The pharmacological and physiological role of cyclic-GMP in vascular smooth muscle relaxation. Am. Rev. Pharmacol. Toxicol. 25:171-191.

12. Owen, M. P., and J. A. Bevan. 1985. Acetylcholine induced endothelium-dependent vasodilation increases as artery diameter decreases in the rabbit ear. Experientia. 41:7-8.

13. Parnavelas, J. G., G. Kelly, and G. Burnstock. 1985. Ultrastructural localization of choline acetyltransferase in vascular endothelial cells in rat brain. Nature (Lond.). 316:724-725.

14. Yasue, K., M. Touyama, M. Shimamoto, M. Kato, S. Tanaka, and F. Akiyama. 1974. Role of autonomic nervous system in the pathogenesis of Prinzmetal's variant form of angina pectoris. Circulation. 50: 534-539.

15. Endo, M., U. Hirosawa, N. Kaneko, K. Hase, Y. Iuone, and S. Konno. 1976. Prinzmetal's variant angina. Coronary arteriogram and ventriculogram during attack induced by metacholine. $N$. Engl. J. Med. 294:252-255.

16. Horio, Y., H. Yasue, M. Rokutanda, N. Nakamura, H. Ogawa, H. Takaoka, K. Matsuyama, and T. Kimura. 1986. Effects of intracoronary injection of acetylcholine on coronary artery diameter. Am. J. Cardiol. 57:984-989. 